\title{
Sensitivity Analysis for a System of Generalized Nonlinear Mixed Quasi Variational Inclusions with H-Monotone Operators
}

\author{
Han-Wen Cao \\ Department of Science, Nanchang Institute of Technology, Nanchang 330099, China \\ Correspondence should be addressed to Han-Wen Cao, chwhappy@163.com \\ Received 20 March 2011; Revised 2 June 2011; Accepted 13 June 2011 \\ Academic Editor: Ya Ping Fang \\ Copyright (C) 2011 Han-Wen Cao. This is an open access article distributed under the Creative \\ Commons Attribution License, which permits unrestricted use, distribution, and reproduction in \\ any medium, provided the original work is properly cited. \\ The existence of the solution for a new system of generalized nonlinear mixed quasi variational \\ inclusions with $\mathrm{H}$-monotone operators is proved by using implicit resolvent technique, and the \\ sensitivity analysis of solution in Hilbert spaces is given. Our results improve and generalize some \\ results of the recent ones.
}

\section{Introduction}

Sensitivity analysis of solution for variational inequalities and variational inclusions has been studied by many authors via quite different technique. (See [1-7] and the reference therein).

In 2004, Agarwal et al. [1] introduced and studied the following problem which is called the system of parametric generalized nonlinear mixed quasi variational inclusions.

Let $\mathscr{l}$ be a real Hilbert space endowed with the product $\langle\cdot, \cdot\rangle$ and norm $\|\cdot\|$, respectively. Let $\Omega$ and $\Lambda$ be two nonempty open subsets of $\mathscr{t}$ in which the parametric $\omega$ and $\lambda$ take values. Let $M: \mathscr{t} \times \Omega \rightarrow 2^{\mathscr{d}}$ and $N$ : $\mathscr{t} \times \Lambda \rightarrow 2^{\mathscr{d}}$ be two maximal monotone mappings with respect to the first argument. $H_{1}, S: \mathscr{l} \times \Omega \rightarrow \mathscr{d}$ and $H_{2}, T: \mathscr{d} \times \Lambda \rightarrow \mathscr{d}$ be nonlinear single-valued mappings. The system of parametric generalized nonlinear mixed quasi variational inclusions problem [1] is to find $(x, y) \in \mathscr{l} \times \mathscr{\ell}$ such that

$$
\begin{aligned}
& 0 \in x-y+\rho\left(H_{1}(y, \omega), S(y, \omega)\right)+\rho M(x, \omega), \\
& 0 \in y-x+\gamma\left(H_{2}(x, \lambda)+T(x, \lambda)\right)+\gamma N(y, \lambda),
\end{aligned}
$$

where $\rho>0$ and $\gamma>0$ are two constants. 
In this paper, we introduce a new system of parametric generalized nonlinear mixed quasi variational inclusions problem.

For each $\omega \in \Omega, \lambda \in \Lambda$, find $x=x(\omega, \lambda), y=y(\omega, \lambda)$ such that

$$
\begin{aligned}
& 0 \in f(x, y, \omega)+\rho_{1}(F(x, y, \omega)+M(x, \omega)) \\
& 0 \in g(x, y, \lambda)+\rho_{2}(G(x, y, \lambda)+N(y, \lambda))
\end{aligned}
$$

where $f, F: \mathscr{l} \times \mathscr{d} \times \Omega \rightarrow \mathscr{H}, g, G: \mathscr{H} \times \mathscr{d} \times \Lambda \rightarrow \mathscr{H}$ are nonlinear single-valued mappings, $M: \mathscr{l} \times \Omega \rightarrow 2^{\mathfrak{l}}, N: \mathscr{d} \times \Lambda \rightarrow 2^{\mathfrak{d}}$ are multivalued mappings, $\rho_{1}>0, \rho_{2}>0$ are constants. By using implicit resolvent equations technique of $H$-monotone operator, the existence of solution is proved and the sensitivity analysis of solution for the problem (1.2) is given. Our results improve and generalize the known results of $[1,2,8,9]$.

\section{Preliminaries}

Let $\mathscr{\ell}$ be a real Hilbert space, $\Omega, \Lambda$ be two nonempty open subsets of $\mathscr{\ell}$.

Definition 2.1 (see [1]). $\quad$ (i) A mapping $T: \mathscr{d} \times \Omega \rightarrow \mathscr{d}$ is said to be monotone with respect to the first argument if

$$
\langle T(x, \omega)-T(y, \omega), x-y\rangle \geq 0, \quad \forall(x, \omega),(y, \omega) \in \mathscr{H} \times \Omega .
$$

(ii) $T$ is said to be $\mathcal{\kappa}$-strongly monotone with respect to the first argument if there exists a constant $\kappa>0$ such that

$$
\langle T(x, \omega)-T(y, \omega), x-y\rangle \geq \kappa\|x-y\|^{2}, \quad \forall(x, \omega),(y, \omega) \in \mathscr{H} \times \Omega .
$$

(iii) $T$ is said to be $(\xi, \eta)$-Lipschitz continuous if there exist $\xi>0, \eta>0$ such that

$$
\left\|T\left(x, \omega_{1}\right)-T\left(y, \omega_{2}\right)\right\| \leq \xi\|x-y\|+\eta\left\|\omega_{1}-\omega_{2}\right\|, \quad \forall\left(x, \omega_{1}\right),\left(y, \omega_{2}\right) \in \mathscr{\ell} \times \Omega .
$$

Definition 2.2 (see [1]). A mapping $F: \mathscr{l} \times \mathscr{H} \times \Omega \rightarrow \mathscr{H}$ is said to be $(\xi, \eta, \zeta)$-Lipschitz continuous if there exist $\xi>0, \eta>0, \zeta>0$ such that

$$
\begin{aligned}
& \left\|F\left(x_{1}, y_{1}, \omega_{1}\right)-F\left(x_{2}, y_{2}, \omega_{2}\right)\right\| \\
& \leq \xi\left\|x_{1}-x_{2}\right\|+\eta\left\|y_{1}-y_{2}\right\|+\zeta\left\|\omega_{1}-\omega_{2}\right\|, \quad \forall\left(x_{1}, y_{1}, \omega_{1}\right),\left(x_{2}, y_{2}, \omega_{2}\right) \in \mathscr{H} \times \mathscr{H} \times \Omega .
\end{aligned}
$$

Definition 2.3 (see [1]). A mapping $F: \mathscr{l} \times \mathscr{l} \times \Omega \rightarrow \mathscr{l}$ is said to be $\alpha$-strongly monotone with respect to $H$ in the first argument if there exists $\alpha>0$ such that

$$
\left\langle F\left(x_{1}, y, \omega\right)-F\left(x_{2}, y, \omega\right), H x_{1}-H x_{2}\right\rangle \geq \alpha\left\|x_{1}-x_{2}\right\|^{2}, \forall\left(x_{1}, y, \omega\right),\left(x_{2}, y, \omega\right) \in \mathscr{d} \times \mathscr{H} \times \Omega
$$


In a similar way, we can define the strong monotonicity of $F$ with respect to $H$ in the second argument.

Definition 2.4 (see [9]). Let $H$ : $\mathscr{t} \times \Omega \rightarrow \mathscr{d}$ be a single-valued mapping and $M$ : $\mathscr{t} \times \Omega \rightarrow$ $2^{\mathscr{H}}$ be a multi-valued mapping. $M$ is said to be $H$-monotone if $M$ is monotone with respect to the first argument and $(H(\cdot, \omega)+\rho M(\cdot, \omega))(\mathscr{\ell})=\mathscr{\ell}$ holds for all $\rho>0$ and $\omega \in \Omega$.

Definition 2.5 (see [9]). Let $H: \mathscr{H} \times \Omega \rightarrow \mathscr{H}$ be a strictly monotone mapping and $M: \mathscr{H} \times \Omega \rightarrow$ $2^{\mathscr{d}}$ be an $H$-monotone mapping. The resolvent operator $J_{M(\cdot, \omega), \rho}^{H}: \mathscr{d} \rightarrow \mathscr{d}$ is defined by

$$
J_{M(\cdot, \omega), \rho}^{H}(u)=(H(\cdot, \omega)+\rho M(\cdot, \omega))^{-1}(u), \quad \forall u \in \mathscr{\ell} .
$$

Lemma 2.6 (see [9]). Let $H_{1}: \mathscr{d e} \times \Omega \rightarrow$ de be $\gamma_{1}$-strongly monotone with respect to the first argument, $H_{2}: \mathscr{d} \times \Lambda \rightarrow \mathscr{d l}$ be $\gamma_{2}$-strongly monotone with respect to the first argument, and $M: \mathscr{d l} \times \Omega \rightarrow 2^{\text {th }}$ be $H_{1}$-monotone, and $N: \mathfrak{d} \times \Lambda \rightarrow 2^{d \mathcal{l}}$ be $\mathrm{H}_{2}$-monotone. Then for any fixed $\omega \in \Omega, \lambda \in \Lambda$, the resolvent operator $J_{M(\cdot, \omega), \rho_{1}}^{H_{1}}$ and $J_{N(\cdot, \lambda), \rho_{2}}^{H_{2}}$ are Lipschitz continuous:

$$
\begin{gathered}
\left\|J_{M(\cdot, \omega), \rho_{1}}^{H_{1}}(u)-J_{M(\cdot, \omega), \rho_{1}}^{H_{1}}(v)\right\| \leq \frac{1}{\gamma_{1}}\|u-v\|, \quad \forall u, v \in \mathscr{L}, \\
\left\|J_{N(\cdot, \lambda), \rho_{2}}^{H_{2}}(u)-J_{N(\cdot, \lambda), \rho_{2}}^{H_{2}}(v)\right\| \leq \frac{1}{\gamma_{2}}\|u-v\|, \quad \forall u, v \in \mathscr{\ell},
\end{gathered}
$$

where $\gamma_{1}>0, \gamma_{2}>0$ are constants.

Lemma 2.7. Let $M: \mathscr{t l} \times \Omega \rightarrow 2^{\mathfrak{d l}}$ be $H_{1}$-monotone and $N: \mathscr{d} \times \Lambda \rightarrow 2^{\mathfrak{d}}$ be $H_{2}$-monotone. For any fixed $(\omega, \lambda) \in \Omega \times \Lambda .(x(\omega, \lambda), y(\omega, \lambda))$ is a solution of (1.2) if and only if

$$
\begin{aligned}
x(\omega, \lambda) & =J_{M(\cdot, \omega), \rho_{1}}^{H_{1}}\left(H_{1}(x(\omega, \lambda), \omega)-f(x(\omega, \lambda), y(\omega, \lambda), \omega)-\rho_{1} F(x(\omega, \lambda), y(\omega, \lambda), \omega)\right) \\
& \triangleq T(x, y, \omega, \lambda) \\
y(\omega, \lambda) & =J_{N(\cdot, \lambda), \rho_{2}}^{H_{2}}\left(H_{2}(y(\omega, \lambda), \lambda)-g(x(\omega, \lambda), y(\omega, \lambda), \lambda)-\rho_{2} G(x(\omega, \lambda), y(\omega, \lambda), \lambda)\right) \\
& \triangleq S(x, y, \omega, \lambda),
\end{aligned}
$$

here $x=x(\omega, \lambda), y=y(\omega, \lambda)$.

Proof. Assume that $(x(\omega, \lambda), y(\omega, \lambda))$ satisfies relations $\left(2.8_{1}\right)$ and $\left(2.8_{2}\right)$, since $J_{M(\cdot, \omega), \rho_{1}}^{H_{1}}=$ $\left(H_{1}(\cdot, \omega)+\rho_{1} M(\cdot, \omega)\right)^{-1}, J_{M(\cdot, \omega), \rho_{2}}^{H_{2}}=\left(H_{2}(\cdot, \omega)+\rho_{2} N(\cdot, \omega)\right)^{-1}$, then $\left(2.8_{1}\right)$ and $\left(2.8_{2}\right)$ holds if and only if

$$
\begin{aligned}
& 0 \in f(x(\omega, \lambda), y(\omega, \lambda), \omega)+\rho_{1} F(x(\omega, \lambda), y(\omega, \lambda), \omega)+\rho_{1} M(x(\omega, \lambda), \omega), \\
& 0 \in g(x(\omega, \lambda), y(\omega, \lambda), \lambda)+\rho_{2} G(x(\omega, \lambda), y(\omega, \lambda), \lambda)+\rho_{2} N(y(\omega, \lambda), \lambda),
\end{aligned}
$$

and hence $(x(\omega, \lambda)), y(\omega, \lambda)$ is a solution of $\left(2.8_{1}\right)$ and $\left(2.8_{2}\right)$. 


\section{Main Results}

Lemma 3.1. Let $T: \mathscr{d} \times \mathscr{d} \rightarrow \mathscr{d}, S: \mathscr{d} \times \mathscr{d} \rightarrow \mathscr{d}$ be two continuous mappings. If there exist $\Theta_{1}$, $\Theta_{2}, 0<\Theta_{1}, \Theta_{2}<1$, such that

$$
\begin{aligned}
& \left\|T\left(x_{1}, y_{1}\right)-T\left(x_{2}, y_{2}\right)\right\|+\left\|S\left(x_{1}, y_{1}\right)-S\left(x_{2}, y_{2}\right)\right\| \\
& \quad \leq \Theta_{1}\left\|x_{1}-x_{2}\right\|+\Theta_{2}\left\|y_{1}-y_{2}\right\|, \quad \forall x_{1}, x_{2}, y_{1}, y_{2} \in \mathcal{H} .
\end{aligned}
$$

then there exist $x^{*}, y^{*} \in \mathscr{d}$ such that

$$
x^{*}=T\left(x^{*}, y^{*}\right), \quad y^{*}=S\left(x^{*}, y^{*}\right) .
$$

Proof. For any $x_{0}, y_{0} \in \mathscr{L}$, let $x_{n+1}=T\left(x_{x}, y_{n}\right), y_{n+1}=S\left(x_{n}, y_{n}\right), n=0,1,2, \ldots$, then by (3.1), for all $x_{1}, x_{2}, \ldots, x_{n}, x_{n+1}, y_{1}, y_{2}, \ldots, y_{n}, y_{n+1} \in \mathscr{d}$, we have

$$
\begin{aligned}
\left\|x_{n+1}-x_{n}\right\|+\left\|y_{n+1}-y_{n}\right\| & \leq \Theta_{1}\left\|x_{n}-x_{n-1}\right\|+\Theta_{2}\left\|y_{n}-y_{n-1}\right\| \\
& \leq \Theta\left(\left\|x_{n}-x_{n-1}\right\|+\left\|y_{n}-y_{n-1}\right\|\right),
\end{aligned}
$$

where $\Theta=\max \left\{\Theta_{1}, \Theta_{2}\right\}$. Let $a=\left\|x_{1}-x_{0}\right\|+\left\|y_{1}-y_{0}\right\|$, then

$$
\begin{aligned}
\left\|x_{n+1}-x_{n}\right\|+\left\|y_{n+1}-y_{n}\right\| & \leq \Theta\left(\left\|x_{n}-x_{n-1}\right\|+\left\|y_{n}-y_{n-1}\right\|\right) \\
& \leq \Theta^{2}\left(\left\|x_{n-1}-x_{n-2}\right\|+\left\|y_{n-1}-y_{n-2}\right\|\right) \\
& \leq \cdots \leq \Theta^{n}\left(\left\|x_{1}-x_{0}\right\|+\left\|y_{1}-y_{0}\right\|\right)=\Theta^{n} a,
\end{aligned}
$$

and hence,

$$
\begin{aligned}
& 0 \leq\left\|x_{n+1}-x_{n}\right\| \leq \Theta^{n} a, \\
& 0 \leq\left\|y_{n+1}-y_{n}\right\| \leq \Theta^{n} a .
\end{aligned}
$$

Since $0<\Theta=\max \left\{\Theta_{1}, \Theta_{2}\right\}<1$, (3.5) implies that $\left\{x_{n}\right\}$ and $\left\{y_{n}\right\}$ are both cauchy sequences. Therefore, there exist $x^{*}, y^{*} \in \mathscr{H}$ such that $x_{n} \rightarrow x^{*}, y_{n} \rightarrow y^{*}(n \rightarrow \infty)$. By continuity of $T$ and $S, x^{*}=T\left(x^{*}, y^{*}\right), y^{*}=S\left(x^{*}, y^{*}\right)$.

Theorem 3.2. Let $H_{1}: \mathscr{d} \times \Omega \rightarrow \mathscr{d}$ be $\left(\xi_{1}, \eta_{1}\right)$-Lipschitz continuous and $H_{2}: \mathscr{d} \times \Lambda \rightarrow \mathscr{d}$ be $\left(\xi_{2}, \eta_{2}\right)$-Lipschitz continuous. Let $f: \mathscr{d} \times \mathscr{d} \times \Omega \rightarrow \mathscr{H}$ be $\left(\xi_{f}, \eta_{f}, \zeta_{f}\right)$-Lipschitz continuous and $\gamma_{f}$-strongly monotone with respect to $H$ in the first argument, $F: \mathscr{d} \times \mathscr{d} \times \Omega \rightarrow \mathscr{H} b e\left(\xi_{F}, \eta_{F}, \zeta_{F}\right)$ Lipschitz continuous, $g: \mathscr{d} \times \mathscr{d} \times \Lambda \rightarrow \mathscr{H}$ be $\left(\xi_{g}, \eta_{g}, \zeta_{g}\right)$-Lipschitz continuous and $\gamma_{g}$-strongly monotone with respect to $H_{2}$ in the second argument, $G: \mathfrak{d} \times \mathscr{d} \times \Lambda \rightarrow \mathscr{d l} b e\left(\xi_{G}, \eta_{G}, \zeta_{G}\right)$-Lipschitz 
continuous. Suppose that $M: \mathscr{d} \times \Omega \rightarrow 2^{\mathscr{d}}$ is $H_{1}$-monotone and $N: \mathscr{d} \times \Lambda \rightarrow 2^{\mathfrak{d}}$ is $\mathrm{H}_{2}$-monotone If

$$
\begin{gathered}
\Theta_{1}=\frac{1}{\gamma_{1}}\left(\sqrt{\xi_{1}^{2}-2 \gamma_{f}+\xi_{f}^{2}}+\rho_{1} \xi_{F}\right)+\frac{\xi_{g}+\rho_{2} \xi_{G}}{\gamma_{2}}<1, \\
\Theta_{2}=\frac{1}{\gamma_{2}}\left(\sqrt{\xi_{2}^{2}-2 \gamma_{g}+\eta_{g}^{2}}+\rho_{2} \eta_{G}\right)+\frac{\eta_{f}+\rho_{1} \eta_{F}}{\gamma_{1}}<1,
\end{gathered}
$$

then, for each $\omega, \lambda \in \Omega \times \Lambda$, the problem (1.2) has an unique solution $\left(x^{*}(\omega, \lambda), y^{*}(\omega, \lambda)\right)$.

Proof. Let $x_{1}=x_{1}(\omega, \lambda), x_{2}=x_{2}(\omega, \lambda), y_{1}=y_{1}(\omega, \lambda), y_{2}=y_{2}(\omega, \lambda)$. By defining $\left(2.8_{1}\right)$ of $T$ and Lemma 2.6, we have

$$
\begin{aligned}
\left\|T\left(x_{1}, y_{1}, \omega, \lambda\right)-T\left(x_{2}, y_{2}, \omega, \lambda\right)\right\| \leq & \frac{1}{r_{1}} \| H_{1}\left(x_{1}, \omega\right)-f\left(x_{1}, y_{1}, \omega\right)-\rho_{1} F\left(x_{1}, y_{1}, \omega\right)-H_{1}\left(x_{2}, \omega\right) \\
& +f\left(x_{2}, y_{2}, \omega\right)+\rho_{1} F\left(x_{2}, y_{2}, \omega\right) \| \\
\leq & \frac{1}{r_{1}}\left\|H_{1}\left(x_{1}, \omega\right)-H_{1}\left(x_{2}, \omega\right)-f\left(x_{1}, y_{1}, \omega\right)+f\left(x_{2}, y_{2}, \omega\right)\right\| \\
& +\frac{1}{r_{1}} \rho_{1}\left\|F\left(x_{1}, y_{1}, \omega\right)-F\left(x_{2}, y_{2}, \omega\right)\right\| .
\end{aligned}
$$

Since $f$ is $\left(\xi_{f}, \eta_{f}, \zeta_{f}\right)$-Lipschitz continuous and $\gamma_{f}$-strongly monotone with respect to $H_{1}$ in the first argument, $F$ is $\left(\xi_{F}, \eta_{F}, \zeta_{F}\right)$-Lipschitz continuous, we have

$$
\begin{aligned}
& \left\|H_{1}\left(x_{1}, \omega\right)-H_{1}\left(x_{2}, \omega\right)-f\left(x_{1}, y_{1}, \omega\right)+f\left(x_{2}, y_{2}, \omega\right)\right\| \\
& \quad \leq\left\|H_{1}\left(x_{1}, \omega\right)-H_{1}\left(x_{2}, \omega\right)-f\left(x_{1}, y_{1}, \omega\right)+f\left(x_{2}, y_{1}, \omega\right)\right\|+\left\|f\left(x_{2}, y_{1}, \omega\right)-f\left(x_{2}, y_{2}, \omega\right)\right\| \\
& \quad \leq \sqrt{\xi_{1}^{2}-2 \gamma_{f}+\xi_{f}^{2}}\left\|x_{1}-x_{2}\right\|+\eta_{f}\left\|y_{1}-y_{2}\right\| \\
& \left\|F\left(x_{1}, y_{1}, \omega\right)-F\left(x_{2}, y_{2}, \omega\right)\right\| \leq \xi_{F}\left\|x_{1}-x_{2}\right\|+\eta_{F}\left\|y_{1}-y_{2}\right\| .
\end{aligned}
$$

Combining (3.7)-(3.9), we have

$$
\begin{aligned}
\left\|T\left(x_{1}, y_{1}, \omega, \lambda\right)-T\left(x_{2}, y_{2}, \omega, \lambda\right)\right\| \leq & \frac{1}{\gamma_{1}}\left(\sqrt{\xi_{1}^{2}-2 \gamma_{f}+\xi_{f}^{2}}+\rho_{1} \xi_{F}\right)\left\|x_{1}-x_{2}\right\| \\
& +\frac{\eta_{f}+\rho_{1} \eta_{F}}{\gamma_{1}}\left\|y_{1}-y_{2}\right\| .
\end{aligned}
$$


By defining $\left(2.8_{2}\right)$ of $S$ and Lemma 2.6, we have

$$
\begin{aligned}
\left\|S\left(x_{1}, y_{1}, \omega, \lambda\right)-S\left(x_{2}, y_{2}, \omega, \lambda\right)\right\| \leq & \frac{1}{\gamma_{2}} \| H_{2}\left(y_{1}, \lambda\right)-g\left(x_{1}, y_{1}, \lambda\right)-\rho_{2} G\left(x_{1}, y_{1}, \lambda\right) \\
& -H_{2}\left(y_{2}, \lambda\right)+g\left(x_{2}, y_{2}, \lambda\right)+\rho_{2} G\left(x_{2}, y_{2}, \lambda\right) \| \\
\leq & \frac{1}{\gamma_{2}}\left\|H_{2}\left(y_{1}, \lambda\right)-H_{2}\left(y_{2}, \lambda\right)-g\left(x_{1}, y_{1}, \lambda\right)+g\left(x_{2}, g_{2}, \lambda\right)\right\| \\
& +\frac{\rho_{2}}{\gamma_{2}}\left\|G\left(x_{1}, y_{1}, \lambda\right)-G\left(x_{2}, y_{2}, \lambda\right)\right\| .
\end{aligned}
$$

Since $g$ is $\left(\xi_{g}, \eta_{g}, \zeta_{g}\right)$-Lipschitz continuous and $\gamma_{g}$-strongly monotone with respect to $\mathrm{H}_{2}$ in the second argument, $G$ is $\left(\xi_{G}, \eta_{G}, \zeta_{G}\right)$-Lipschitz continuous. We have

$$
\begin{aligned}
& \left\|H_{2}\left(y_{1}, \lambda\right)-H_{2}\left(y_{2}, \lambda\right)-g\left(x_{1}, y_{1}, \lambda\right)+g\left(x_{2}, y_{2}, \lambda\right)\right\| \\
& \leq\left\|H_{2}\left(y_{1}, \lambda\right)-H_{2}\left(y_{2}, \lambda\right)-g\left(x_{1}, y_{1}, \lambda\right)+g\left(x_{1}, y_{2}, \lambda\right)\right\|+\left\|g\left(x_{1}, y_{2}, \lambda\right)-g\left(x_{2}, y_{2}, \lambda\right)\right\| \\
& \leq \sqrt{\xi_{2}^{2}-2 \gamma_{g}+\eta_{g}^{2}}\left\|y_{1}-y_{2}\right\|+\xi_{g}\left\|x_{1}-x_{2}\right\|, \\
& \left\|G\left(x_{1}, y_{1}, \lambda\right)-G\left(x_{2}, y_{2}, \lambda\right)\right\| \leq \xi_{G}\left\|x_{1}-x_{2}\right\|+\eta_{G}\left\|y_{1}-y_{2}\right\|
\end{aligned}
$$

Combining (3.11)-(3.13), we have

$$
\begin{aligned}
\left\|S\left(x_{1}, y_{1}, \omega, \lambda\right)-S\left(x_{2}, y_{2}, \omega, \lambda\right)\right\| \leq & \frac{1}{\gamma_{2}}\left(\sqrt{\xi_{2}^{2}-2 \gamma_{g}+\eta_{g}^{2}}+\rho_{2} \eta_{G}\right)\left\|y_{1}-y_{2}\right\| \\
& +\frac{\xi_{g}+\rho_{2} \xi_{G}}{\gamma_{2}}\left\|x_{1}-x_{2}\right\| .
\end{aligned}
$$

By (3.10) and (3.14), we have

$$
\begin{aligned}
&\left\|T\left(x_{1}, y_{1}, \omega, \lambda\right)-T\left(x_{2}, y_{2}, \omega, \lambda\right)\right\|+\left\|S\left(x_{1}, y_{1}, \omega, \lambda\right)-S\left(x_{2}, y_{2}, \omega, \lambda\right)\right\| \\
& \leq {\left[\frac{1}{\gamma_{1}}\left(\sqrt{\xi_{1}^{2}-2 \gamma_{f}+\xi_{f}^{2}}+\rho_{1} \xi_{F}\right)+\frac{\xi_{g}+\rho_{2} \xi_{G}}{\gamma_{2}}\right]\left\|x_{1}-x_{2}\right\| } \\
&+\left[\frac{1}{\gamma_{2}}\left(\sqrt{\xi_{2}^{2}-2 \gamma_{g}+\eta_{g}^{2}}+\rho_{2} \eta_{G}\right)+\frac{\eta_{f}+\rho_{1} \eta_{F}}{\gamma_{1}}\right]\left\|y_{1}-y_{2}\right\| \\
&= \Theta_{1}\left\|x_{1}-x_{2}\right\|+\Theta_{2}\left\|y_{1}-y_{2}\right\| .
\end{aligned}
$$

By (3.6) and Lemma 3.1, there exist $x^{*}=x^{*}(\omega, \lambda), y^{*}=y^{*}(\omega, \lambda) .\left(x^{*}, y^{*}\right)$ such that $x^{*}=$ $T\left(x^{*}, y^{*}, \omega, \lambda\right), y^{*}=S\left(x^{*}, y^{*}, \omega, \lambda\right)$. By Lemma $2.7,\left(x^{*}, y^{*}\right)$ is a solution of (1.2). From (3.15), we easily see that the solution of (1.2) is unique. 
Assumption 1. For implicit resolvent operator $J_{M(\cdot, \omega), \rho_{1}{ }^{\prime}}^{H_{1}} J_{N(\cdot, \lambda), \rho_{2}{ }^{\prime}}^{H_{2}}$, there are two constants $\xi>0$ and $\eta>0$ such that

$$
\begin{aligned}
& \left\|J_{M(\cdot, \omega), \rho_{1}}^{H_{1}}(u)-J_{M(\cdot, \bar{\omega}), \rho_{1}}^{H_{1}}(u)\right\| \leq \xi\|\omega-\bar{\omega}\|, \\
& \left\|J_{N(\cdot, \lambda), \rho_{2}}^{H_{2}}(v)-J_{N(\cdot, \bar{\lambda}), \rho_{2}}^{H_{2}}(v)\right\| \leq \eta\|\lambda-\bar{\lambda}\|, \quad \forall u, v \in \mathscr{H} .
\end{aligned}
$$

Theorem 3.3. Suppose that the mappings $H_{1}, f, F, M, H_{2}, g, G$, and $N$ are the same as in Theorem 3.2, and for any fixed $x, y \in \mathcal{d}$, the mappings $\omega \rightarrow H_{1}(x, \omega), \omega \rightarrow f(x, y, \omega), \omega \rightarrow$ $F(x, y, \omega), \lambda \rightarrow H_{2}(y, \lambda), \lambda \rightarrow g(x, y, \lambda), \lambda \rightarrow G(x, y, \lambda)$ are continuous (or Lipschitz continuous.) If Assumption 1 and condition (3.6) hold, then the solution $(x(\omega, \lambda), y(\omega, \lambda))$ for the problem (1.2) is continuous (or Lipschitz continuous).

Proof. Suppose that $\omega, \bar{\omega} \in \Omega, \lambda, \bar{\lambda} \in \Lambda$ such that $\omega \rightarrow \bar{\omega}, \lambda \rightarrow \bar{\lambda}$. From Theorem 3.2, we know that the problem (1.2) has solution $(x(\omega, \lambda), y(\omega, \lambda)), x(\bar{\omega}, \bar{\lambda}), y(\bar{\omega}, \bar{\lambda})),(x(\bar{\omega}, \lambda), y(\bar{\omega}, \lambda))$ and $(x(\omega, \bar{\lambda}), y(\omega, \bar{\lambda}))$.

(A) Estimate $\|x(\omega, \lambda)-x(\bar{\omega}, \lambda)\|$ and $\|y(\omega, \lambda)-y(\bar{\omega}, \lambda)\|$. By Lemma 2.7 , we have

$$
\begin{aligned}
x(\omega, \lambda) & =J_{M(\cdot, \omega), \rho_{1}}^{H_{1}}\left(H_{1}(x(\omega, \lambda), \omega)-f(x(\omega, \lambda), y(\omega, \lambda), \omega)-\rho_{1} F(x(\omega, \lambda), y(\omega, \lambda), \omega)\right) \\
& \triangleq J_{M(\cdot, \omega), \rho_{1}}^{H_{1}}(p), \\
x(\bar{\omega}, \lambda) & =J_{M(\cdot, \bar{\omega}), \rho_{1}}^{H_{1}}\left(H_{1}(x(\bar{\omega}, \lambda), \bar{\omega})-f(x(\bar{\omega}, \lambda), y(\bar{\omega}, \lambda), \bar{\omega})-\rho_{1} F(x(\bar{\omega}, \lambda), y(\bar{\omega}, \lambda), \bar{\omega})\right) \\
& \triangleq J_{M(\cdot, \bar{\omega}), \rho_{1}}^{H_{1}}(q) .
\end{aligned}
$$

It follows from Assumption 1, Lemmas 2.6 and 2.7 that

$$
\begin{aligned}
& \|x(\omega, \lambda)-x(\bar{\omega}, \lambda)\|=\left\|J_{M(\cdot, \omega), \rho_{1}}^{H_{1}}(p)-J_{M(\cdot, \bar{\omega}), \rho_{1}}^{H_{1}}(q)\right\| \\
& \quad \leq\left\|J_{M(\cdot, \omega), \rho_{1}}^{H_{1}}(p)-J_{M(\cdot, \omega), \rho_{1}}^{H_{1}}(q)\right\|+\left\|J_{M(\cdot, \omega), \rho_{1}}^{H_{1}}(q)-J_{M(\cdot, \bar{\omega}), \rho_{1}}^{H_{1}}(q)\right\| \leq \frac{1}{\gamma_{1}}\|p-q\|+\xi\|\omega-\bar{\omega}\| .
\end{aligned}
$$

Since $f$ is $\left(\xi_{f}, \eta_{f}, \zeta_{f}\right)$-Lipschitz continuous, $\gamma_{f}$-strongly monotone with respect to $H_{1}$ in the first argument and $F$ is $\left(\xi_{F}, \eta_{F}, \zeta_{F}\right)$-Lipschitz continuous, we conclude

$$
\begin{aligned}
\|p-q\|= & \| H_{1}(x(\omega, \lambda), \omega)-f(x(\omega, \lambda), y(\omega, \lambda), \omega)-\rho_{1} F(x(\omega, \lambda), y(\omega, \lambda), \omega) \\
& -H_{1}(x(\bar{\omega}, \lambda), \bar{\omega})+f(x(\bar{\omega}, \lambda), y(\bar{\omega}, \lambda), \bar{\omega})+\rho_{1} F(x(\bar{\omega}, \lambda), y(\bar{\omega}, \lambda), \bar{\omega}) \| \\
\leq & \left\|H_{1}(x(\omega, \lambda), \omega)-H_{1}(x(\bar{\omega}, \lambda), \omega)-f(x(\omega, \lambda), y(\omega, \lambda), \omega)+f(x(\bar{\omega}, \lambda), y(\omega, \lambda), \omega)\right\| \\
& +\left\|H_{1}(x(\bar{\omega}, \lambda), \omega)-H_{1}(x(\bar{\omega}, \lambda), \bar{\omega})\right\| \\
& +\|f(x(\bar{\omega}, \lambda), y(\omega, \lambda), \omega)-f(x(\bar{\omega}, \lambda), y(\bar{\omega}, \lambda), \omega)\|
\end{aligned}
$$




$$
\begin{aligned}
& +\|f(x(\bar{\omega}, \lambda), y(\bar{\omega}, \lambda), \omega)-f(x(\bar{\omega}, \lambda), y(\bar{\omega}, \lambda), \bar{\omega})\| \\
& +\rho_{1}\|F(x(\omega, \lambda), y(\omega, \lambda), \omega)-F(x(\bar{\omega}, \lambda), y(\bar{\omega}, \lambda), \omega)\| \\
& +\rho_{1}\|F(x(\bar{\omega}, \lambda), y(\bar{\omega}, \lambda), \omega)-F(x(\bar{\omega}, \lambda), y(\bar{\omega}, \lambda), \bar{\omega})\| \\
& \leq \sqrt{\xi_{1}^{2}-2 \gamma_{f}+\xi_{f}^{2}}\|x(\omega, \lambda)-x(\bar{\omega}, \lambda)\|+\left\|H_{1}(x(\bar{\omega}, \lambda), \omega)-H_{1}(x(\bar{\omega}, \lambda), \bar{\omega})\right\| \\
& +\eta_{f}\|y(\omega, \lambda)-y(\bar{\omega}, \lambda)\|+\|f(x(\bar{\omega}, \lambda), y(\bar{\omega}, \lambda), \omega)-f(x(\bar{\omega}, \lambda), y(\bar{\omega}, \lambda), \bar{\omega})\| \\
& +\rho_{1} \xi_{F}\|x(\omega, \lambda)-x(\bar{\omega}, \lambda)\|+\rho_{1} \eta_{F}\|y(\omega, \lambda)-y(\bar{\omega}, \lambda)\| \\
& +\rho_{1}\|F(x(\bar{\omega}, \lambda), y(\bar{\omega}, \lambda), \omega)-F(x(\bar{\omega}, \lambda), y(\bar{\omega}, \lambda), \bar{\omega})\| .
\end{aligned}
$$

It follows from (3.18) and (3.19) that

$$
\begin{aligned}
\|x(\omega, \lambda)-x(\bar{\omega}, \lambda)\| \leq & \theta_{1}\|x(\omega, \lambda)-x(\bar{\omega}, \lambda)\|+\frac{1}{\gamma_{1}}\left(\eta_{f}+\rho_{1} \eta_{F}\right)\|y(\omega, \lambda)-y(\bar{\omega}, \lambda)\| \\
& +\frac{1}{\gamma_{1}}\left\|H_{1}(x(\bar{\omega}, \lambda), \omega)-H_{1}(x(\bar{\omega}, \lambda), \bar{\omega})\right\| \\
& +\frac{1}{\gamma_{1}}\|f(x(\bar{\omega}, \lambda), y(\bar{\omega}, \lambda), \omega)-f(x(\bar{\omega}, \lambda), y(\bar{\omega}, \lambda), \bar{\omega})\| \\
& +\frac{\rho_{1}}{r_{1}}\|F(x(\bar{\omega}, \lambda), y(\bar{\omega}, \lambda), \omega)-F(x(\bar{\omega}, \lambda), y(\bar{\omega}, \lambda), \bar{\omega})\|+\xi\|\omega-\bar{\omega}\|,
\end{aligned}
$$

that is,

$$
\begin{aligned}
\|x(\omega, \lambda)-x(\bar{\omega}, \lambda)\| \leq \frac{\eta_{f}+\rho_{1} \eta_{F}}{\left(1-\theta_{1}\right) \gamma_{1}} \| & y(\omega, \lambda)-y(\bar{\omega}, \lambda) \| \\
& +\frac{1}{1-\theta_{1}}\left\{\frac{1}{\gamma_{1}}\left\|H_{1}(x(\bar{\omega}, \lambda), \omega)-H_{1}(x(\bar{\omega}, \lambda), \bar{\omega})\right\|\right. \\
& +\frac{1}{\gamma_{1}}\|f(x(\bar{\omega}, \lambda), y(\bar{\omega}, \lambda), \omega)-f(x(\bar{\omega}, \lambda), y(\bar{\omega}, \lambda), \bar{\omega})\| \\
& +\frac{\rho_{1}}{\gamma_{1}}\|F(x(\bar{\omega}, \lambda), y(\bar{\omega}, \lambda), \omega)-F(x(\bar{\omega}, \lambda), y(\bar{\omega}, \lambda), \bar{\omega})\| \\
& +\xi\|\omega-\bar{\omega}\|\},
\end{aligned}
$$

where

$$
\theta_{1}=\frac{1}{\gamma_{1}}\left(\sqrt{\xi_{1}^{2}-2 \gamma_{f}+\xi_{f}^{2}}+\rho_{1} \xi_{F}\right)
$$


Journal of Applied Mathematics

By Lemma 2.7, we have

$$
\begin{aligned}
y(\omega, \lambda) & =J_{N(\cdot, \lambda) \rho_{2}}^{H_{2}}\left(H_{2}(y(\omega, \lambda), \lambda)-g(x(\omega, \lambda), y(\omega, \lambda), \lambda)-\rho_{2} G(x(\omega, \lambda), y(\omega, \lambda), \lambda)\right) \\
& \triangleq J_{N(\cdot, \lambda), \rho_{2}}^{H_{2}}(s), \\
y(\bar{\omega}, \lambda) & =J_{N(\cdot, \lambda), \rho_{2}}^{H_{2}}\left(H_{2}(y(\bar{\omega}, \lambda), \lambda)-g(x(\bar{\omega}, \lambda), y(\bar{\omega}, \lambda), \lambda)-\rho_{2} G(x(\bar{\omega}, \lambda), y(\bar{\omega}, \lambda), \lambda)\right) \\
& \triangleq J_{N(\cdot, \lambda), \rho_{2}}^{H_{2}}(t) .
\end{aligned}
$$

It follows from Assumption 1, Lemmas 2.6 and 2.7 that

$$
\|y(\omega, \lambda)-y(\bar{\omega}, \lambda)\|=\left\|J_{N(\cdot, \lambda), \rho_{2}}^{H_{2}}(s)-J_{N(\cdot, \lambda), \rho_{2}}^{H_{2}}(t)\right\| \leq \frac{1}{\gamma_{2}}\|s-t\| .
$$

Since $g$ is $\left(\xi_{g}, \eta_{g}, \zeta_{g}\right)$-Lipschitz continuous and $\gamma_{g}$-strongly monotone with respect to $\mathrm{H}_{2}$ in the second argument and $G$ is $\left(\xi_{G}, \eta_{G}, \zeta_{G}\right)$-Lipschitz continuous,we conclude

$$
\begin{aligned}
\|s-t\|= & \| H_{2}(y(\omega, \lambda), \lambda)-g(x(\omega, \lambda), y(\omega, \lambda), \lambda)-\rho_{2} G(x(\omega, \lambda), y(\omega, \lambda), \lambda) \\
& -H_{2}(y(\bar{\omega}, \lambda), \lambda)+g(x(\bar{\omega}, \lambda), y(\bar{\omega}, \lambda), \lambda)+\rho_{2} G(x(\bar{\omega}, \lambda), y(\bar{\omega}, \lambda), \lambda) \| \\
\leq & \left\|H_{2}(y(\omega, \lambda), \lambda)-H_{2}(y(\bar{\omega}, \lambda), \lambda)-g(x(\omega, \lambda), y(\omega, \lambda), \lambda)+g(x(\omega, \lambda), y(\bar{\omega}, \lambda), \lambda)\right\| \\
& +\|g(x(\omega, \lambda), y(\bar{\omega}, \lambda), \lambda)-g(x(\bar{\omega}, \lambda), y(\bar{\omega}, \lambda), \lambda)\| \\
& +\rho_{2}\|G(x(\omega, \lambda), y(\omega, \lambda), \lambda)-G(x(\bar{\omega}, \lambda), y(\bar{\omega}, \lambda), \lambda)\| \\
\leq & \sqrt{\xi_{2}^{2}-2 \gamma_{g}+\eta_{g}^{2}}\|y(\omega, \lambda)-y(\bar{\omega}, \lambda)\|+\xi_{g}\|x(\omega, \lambda)-x(\bar{\omega}, \lambda)\| \\
& +\rho_{2} \xi G\|x(\omega, \lambda)-x(\bar{\omega}, \lambda)\|+\rho_{2} \eta_{G}\|y(\omega, \lambda)-y(\bar{\omega}, \lambda)\| .
\end{aligned}
$$

It follows from (3.23) and (3.24) that

$$
\begin{aligned}
\|y(\omega, \lambda)-y(\bar{\omega}, \lambda)\| \leq & \frac{1}{\gamma_{2}}\left(\sqrt{\xi_{2}^{2}-2 \gamma_{g}+\eta_{g}^{2}}+\rho_{2} \eta_{G}\right)\|y(\omega, \lambda)-y(\bar{\omega}, \lambda)\| \\
& +\frac{1}{\gamma_{2}}\left(\xi_{g}+\rho_{2} \xi_{G}\right)\|x(\omega, \lambda)-x(\bar{\omega}, \lambda)\| .
\end{aligned}
$$

That is,

$$
\|y(\omega, \lambda)-y(\bar{\omega}, \lambda)\| \leq \frac{1}{1-\theta_{2}} \frac{1}{\gamma_{2}}\left(\xi_{g}+\rho_{2} \xi_{G}\right)\|x(\omega, \lambda)-x(\bar{\omega}, \lambda)\|,
$$


where

$$
\theta_{2}=\frac{1}{\gamma_{2}}\left(\sqrt{\xi_{2}^{2}-2 \gamma_{g}+\eta_{g}^{2}}+\rho_{2} \eta_{G}\right)
$$

By combining (3.21) and (3.26), we derive

$$
\begin{aligned}
\|x(\omega, \lambda)-x(\bar{\omega}, \lambda)\| \leq & \frac{\left(\eta_{f}+\rho_{1} \eta_{F}\right)\left(\xi_{g}+\rho_{2} \xi_{G}\right)}{\left(1-\theta_{1}\right)\left(1-\theta_{2}\right) \gamma_{1} \gamma_{2}}\|x(\omega, \lambda)-x(\bar{\omega}, \lambda)\| \\
+\frac{1}{1-\theta_{1}}\{ & \frac{1}{\gamma_{1}}\left\|H_{1}(x(\bar{\omega}, \lambda), \omega)-H_{1}(x(\bar{\omega}, \lambda), \bar{\omega})\right\| \\
& +\frac{1}{\gamma_{1}}\|f(x(\bar{\omega}, \lambda), y(\bar{\omega}, \lambda), \omega)-f(x(\bar{\omega}, \lambda), y(\bar{\omega}, \lambda), \bar{\omega})\| \\
& +\frac{\rho_{1}}{\gamma_{1}}\|F(x(\bar{\omega}, \lambda), y(\bar{\omega}, \lambda), \omega)-F(x(\bar{\omega}, \lambda), y(\bar{\omega}, \lambda), \bar{\omega})\| \\
& +\xi\|\omega-\bar{\omega}\| s\} .
\end{aligned}
$$

That is,

$$
\begin{aligned}
\|x(\omega, \lambda)-x(\bar{\omega}, \lambda)\| \leq \frac{1}{1-\kappa} \frac{1}{1-\theta_{1}}\{ & \frac{1}{\gamma_{1}}\left\|H_{1}(x(\bar{\omega}, \lambda), \omega)-H_{1}(x(\bar{\omega}, \lambda), \bar{\omega})\right\| \\
& +\frac{1}{\gamma_{1}}\|f(x(\bar{\omega}, \lambda), y(\bar{\omega}, \lambda), \omega)-f(x(\bar{\omega}, \lambda), y(\bar{\omega}, \lambda), \bar{\omega})\| \\
& +\frac{\rho_{1}}{\gamma_{1}}\|F(x(\bar{\omega}, \lambda), y(\bar{\omega}, \lambda), \omega)-F(x(\bar{\omega}, \lambda), y(\bar{\omega}, \lambda), \bar{\omega})\| \\
& +\xi\|\omega-\bar{\omega}\|\},
\end{aligned}
$$

$$
\begin{aligned}
&\|y(\omega, \lambda)-y(\bar{\omega}, \lambda)\| \leq \frac{\xi_{g}+\rho_{2} \xi_{G}}{\left(1-\theta_{2}\right) \gamma_{2}} \frac{1}{1-\kappa} \frac{1}{1-\theta_{1}}\left\{\frac{1}{r_{1}}\left\|H_{1}(x(\bar{\omega}, \lambda), \omega)-H_{1}(x(\bar{\omega}, \lambda), \bar{\omega})\right\|\right. \\
&+ \frac{1}{r_{1}} \| f(x(\bar{\omega}, \lambda), y(\bar{\omega}, \lambda), \omega) \\
& f(x(\bar{\omega}, \lambda), y(\bar{\omega}, \lambda), \bar{\omega}) \| \\
&+\frac{\rho_{1}}{r_{1}} \| F(x(\bar{\omega}, \lambda), y(\bar{\omega}, \lambda), \omega) \\
&-F(x(\bar{\omega}, \lambda), y(\bar{\omega}, \lambda), \bar{\omega}) \| \\
&+\|\xi \omega-\bar{\omega}\|\},
\end{aligned}
$$


Journal of Applied Mathematics

where

$$
\kappa=\frac{\left(\eta_{f}+\rho_{1} \eta_{F}\right)\left(\xi_{g}+\rho_{2} \xi_{G}\right)}{\left(1-\theta_{1}\right)\left(1-\theta_{2}\right) \gamma_{1} \gamma_{2}} .
$$

By (3.6),

$$
1-\theta_{1}>\frac{1}{\gamma_{2}}\left(\xi_{g}+\rho_{2} \xi_{G}\right), \quad 1-\theta_{2}>\frac{1}{\gamma_{1}}\left(\eta_{f}+\rho_{1} \eta_{F}\right),
$$

and hence

$$
0<\frac{\left(\xi_{g}+\rho_{2} \xi_{G}\right)\left(\eta_{f}+\rho_{1} \eta_{F}\right)}{\left(1-\theta_{1}\right)\left(1-\theta_{2}\right) \gamma_{1} \gamma_{2}}<1, \text { that is, } 0<\kappa<1
$$

(B) Estimate $\|y(\bar{\omega}, \bar{\lambda})-y(\bar{\omega}, \lambda)\|$ and $\|x(\bar{\omega}, \bar{\lambda})-x(\bar{\omega}, \lambda)\|$.

By Lemma 2.7, we have

$$
\begin{aligned}
y(\bar{\omega}, \bar{\lambda}) & =J_{N(,, \bar{\lambda}), \rho_{2}}^{H_{2}}\left(H_{2}(y(\bar{\omega}, \bar{\lambda}), \bar{\lambda})-g(x(\bar{\omega}, \bar{\lambda}), y(\bar{\omega}, \bar{\lambda}), \bar{\lambda})-\rho_{2} G(x(\bar{\omega}, \bar{\lambda}), y(\bar{\omega}, \bar{\lambda}), \bar{\lambda})\right) \\
& =J_{N(\cdot, \bar{\lambda}), \rho_{2}}^{H_{2}}(m), \\
y(\bar{\omega}, \bar{\lambda}) & =J_{N(\cdot, \lambda), \rho_{2}}^{H_{2}}\left(H_{2}(y(\bar{\omega}, \lambda), \lambda)-g(x(\bar{\omega}, \lambda), y(\bar{\omega}, \lambda), \lambda)-\rho_{2} G(x(\bar{\omega}, \lambda), y(\bar{\omega}, \lambda), \lambda)\right) \\
& =J_{N(\cdot, \lambda), \rho_{2}}^{H_{2}}(n) .
\end{aligned}
$$

It follows from Assumption 1, Lemmas 2.6 and 2.7 that

$$
\begin{aligned}
\|y(\bar{\omega}, \bar{\lambda})-y(\bar{\omega}, \lambda)\|= & \left\|J_{N(\cdot, \bar{\lambda}), \rho_{2}}^{H_{2}}(m)-J_{N(\cdot, \lambda), \rho_{2}}^{H_{2}}(n)\right\| \\
& \leq\left\|J_{N(\cdot, \bar{\lambda}), \rho_{2}}^{H_{2}}(m)-J_{N(\cdot, \bar{\lambda}), \rho_{2}}^{H_{2}}(n)\right\|+\left\|J_{N\left(\cdot, \bar{\lambda}, \rho_{2}\right.}^{H_{2}}(n)-J_{N(\cdot, \lambda), \rho_{2}}^{H_{2}}(n)\right\| \\
& \leq \frac{1}{\gamma_{2}}\|m-n\|+\eta\|\lambda-\bar{\lambda}\| .
\end{aligned}
$$

Since $g$ is $\left(\xi_{g}, \eta_{g}, \zeta_{g}\right)$-Lipschitz continuous and $\gamma_{g}$-strongly monotone with respect to $\mathrm{H}_{2}$ in the second argument and $G$ is $\left(\xi_{G}, \eta_{G}, \zeta_{G}\right)$-Lipschitz continuous,we conclude

$$
\begin{aligned}
\|m-n\|= & \| H_{2}(y(\bar{\omega}, \bar{\lambda}), \bar{\lambda})-g(x(\bar{\omega}, \bar{\lambda}), y(\bar{\omega}, \bar{\lambda}), \bar{\lambda})-\rho_{2} G(x(\bar{\omega}, \bar{\lambda}), y(\bar{\omega}, \bar{\lambda}), \bar{\lambda}) \\
& -H_{2}(y(\bar{\omega}, \lambda), \lambda)+g(x(\bar{\omega}, \lambda), y(\bar{\omega}, \lambda), \lambda)+\rho_{2} G(x(\bar{\omega}, \lambda), y(\bar{\omega}, \lambda), \lambda) \| \\
\leq & \| H_{2}(y(\bar{\omega}, \bar{\lambda}), \bar{\lambda})-H_{2}(y(\bar{\omega}, \lambda), \bar{\lambda}) \\
& -g(x(\bar{\omega}, \bar{\lambda}), y(\bar{\omega}, \bar{\lambda}), \bar{\lambda})-g(x(\bar{\omega}, \bar{\lambda}), y(\bar{\omega}, \lambda), \bar{\lambda}) \|
\end{aligned}
$$




$$
\begin{aligned}
& +\left\|H_{2}(y(\bar{\omega}, \lambda), \bar{\lambda})-H_{2}(y(\bar{\omega}, \lambda), \lambda)\right\| \\
& +\|g(x(\bar{\omega}, \bar{\lambda}), y(\bar{\omega}, \lambda), \bar{\lambda})-g(x(\bar{\omega}, \lambda), y(\bar{\omega}, \lambda), \bar{\lambda})\| \\
& +\|g(x(\bar{\omega}, \lambda), y(\bar{\omega}, \lambda), \bar{\lambda})-g(x(\bar{\omega}, \lambda), y(\bar{\omega}, \lambda), \lambda)\| \\
& +\rho_{2}\|G(x(\bar{\omega}, \bar{\lambda}), y(\bar{\omega}, \bar{\lambda}), \bar{\lambda})-G(x(\bar{\omega}, \lambda), y(\bar{\omega}, \lambda), \bar{\lambda})\| \\
& +\rho_{2}\|G(x(\bar{\omega}, \lambda), y(\bar{\omega}, \lambda), \bar{\lambda})-G(x(\bar{\omega}, \lambda), y(\bar{\omega}, \lambda), \lambda)\| \\
& \leq \\
& \sqrt{\xi_{2}^{2}-2 \gamma_{g}+\eta_{g}^{2}}\|y(\bar{\omega}, \bar{\lambda})-y(\bar{\omega}, \lambda)\|+\xi_{g}\|x(\bar{\omega}, \bar{\lambda})-x(\bar{\omega}, \lambda)\| \\
& +\rho_{2}\left(\xi \xi_{G}\|x(\bar{\omega}, \bar{\lambda})-x(\bar{\omega}, \lambda)\|+\eta_{G}\|y(\bar{\omega}, \bar{\lambda})-y(\bar{\omega}, \lambda)\|\right) \\
& +\|g(x(\bar{\omega}, \lambda), y(\bar{\omega}, \lambda), \bar{\lambda})-g(x(\bar{\omega}, \lambda), y(\bar{\omega}, \lambda), \lambda)\| \\
& +\left\|H_{2}(y(\bar{\omega}, \lambda), \bar{\lambda})-H_{2}(y(\bar{\omega}, \lambda), \lambda)\right\| \\
& +\rho_{2}\|G(x(\bar{\omega}, \lambda), y(\bar{\omega}, \lambda), \bar{\lambda})-G(x(\bar{\omega}, \lambda), y(\bar{\omega}, \lambda), \lambda)\| .
\end{aligned}
$$

It follows from (3.33) and (3.34) that

$$
\begin{gathered}
\|y(\bar{\omega}, \bar{\lambda})-y(\bar{\omega}, \lambda)\| \leq \frac{\xi_{g}+\rho_{2} \xi_{G}}{\left(1-\theta_{2}\right) \gamma_{2}}\|x(\bar{\omega}, \bar{\lambda})-x(\bar{\omega}, \lambda)\| \\
+\frac{1}{1-\theta_{2}}\left\{\frac{1}{\gamma_{2}}\left\|H_{2}(y(\bar{\omega}, \lambda), \bar{\lambda})-H_{2}(y(\bar{\omega}, \lambda), \lambda)\right\|\right. \\
+\frac{1}{\gamma_{2}} \| g(x(\bar{\omega}, \lambda), y(\bar{\omega}, \lambda), \bar{\lambda}) \\
-g(x(\bar{\omega}, \lambda), y(\bar{\omega}, \lambda), \lambda) \| \\
+\frac{\rho_{2}}{\gamma_{2}} \| G(x(\bar{\omega}, \lambda), y(\bar{\omega}, \lambda), \bar{\lambda}) \\
-G(x(\bar{\omega}, \lambda), y(\bar{\omega}, \lambda), \lambda) \| \\
+\eta\|\lambda-\bar{\lambda}\|\},
\end{gathered}
$$

where $\theta_{2}$ defined by $(* *)$. 
Journal of Applied Mathematics

By Lemma 2.7, we have

$$
\begin{gathered}
x(\bar{\omega}, \bar{\lambda})=J_{M(\cdot, \bar{\omega}), \rho_{1}}^{H_{1}}\left(H_{1}(x(\bar{\omega}, \bar{\lambda}), \bar{\omega})-f(x(\bar{\omega}, \bar{\lambda}), y(\bar{\omega}, \bar{\lambda}), \bar{\omega})-\rho_{1} F(x(\bar{\omega}, \bar{\lambda}), y(\bar{\omega}, \bar{\lambda}), \bar{\omega})\right), \\
x(\bar{\omega}, \lambda)=J_{M(\cdot, \bar{\omega}), \rho_{1}}^{H_{1}}\left(H_{1}(x(\bar{\omega}, \lambda), \bar{\omega})-f(x(\bar{\omega}, \lambda), y(\bar{\omega}, \lambda), \bar{\omega})-\rho_{1} F(x(\bar{\omega}, \lambda), y(\bar{\omega}, \lambda), \bar{\omega})\right) .
\end{gathered}
$$

As the proof of (3.25), we have

$$
\begin{aligned}
\|x(\bar{\omega}, \bar{\lambda})-x(\bar{\omega}, \lambda)\| \leq & \left(\frac{1}{\gamma_{1}} \sqrt{\xi_{1}^{2}-2 \gamma_{f}+\xi_{f}^{2}}+\rho_{1} \xi_{F}\right)\|x(\bar{\omega}, \bar{\lambda})-x(\bar{\omega}, \lambda)\| \\
& +\frac{1}{\gamma_{1}}\left(\eta_{f}+\rho_{1} \eta_{F}\right)\|y(\bar{\omega}, \bar{\lambda})-y(\bar{\omega}, \lambda)\|
\end{aligned}
$$

that is,

$$
\|x(\bar{\omega}, \bar{\lambda})-x(\bar{\omega}, \lambda)\| \leq \frac{\eta_{f}+\rho_{1} \eta_{F}}{\left(1-\theta_{1}\right) \gamma_{1}}\|y(\bar{\omega}, \bar{\lambda})-y(\bar{\omega}, \lambda)\|,
$$

where $\theta_{1}$ is defined by $(*)$.

Therefore

$$
\begin{aligned}
\|y(\bar{\omega}, \bar{\lambda})-y(\bar{\omega}, \lambda)\| \leq \frac{1}{1-\kappa} \frac{1}{1-\theta_{2}}\left\{\frac{1}{\gamma_{2}}\left\|H_{2}(y(\bar{\omega}, \lambda), \bar{\lambda})-H_{2}(y(\bar{\omega}, \lambda), \lambda)\right\|\right. \\
+\frac{1}{\gamma_{2}} \| g(x(\bar{\omega}, \lambda), y(\bar{\omega}, \lambda), \bar{\lambda}) \\
\quad-g(x(\bar{\omega}, \lambda), y(\bar{\omega}, \lambda), \lambda) \| \\
+\frac{\rho_{2}}{\gamma_{2}} \| G(x(\bar{\omega}, \lambda), y(\bar{\omega}, \lambda), \bar{\lambda}) \\
-G(x(\bar{\omega}, \lambda), y(\bar{\omega}, \lambda), \lambda) \| \\
+\eta\|\lambda-\bar{\lambda}\|\}
\end{aligned}
$$




$$
\begin{aligned}
&\|x(\bar{\omega}, \bar{\lambda})-x(\bar{\omega}, \lambda)\| \leq \frac{\eta_{f}+\rho_{1} \eta_{F}}{\left(1-\theta_{1}\right) \gamma_{1}} \frac{1}{1-\mathcal{\kappa}} \frac{1}{1-\theta_{2}}\left\{\frac{1}{\gamma_{2}}\left\|H_{2}(y(\bar{\omega}, \lambda), \bar{\lambda})-H_{2}(y(\bar{\omega}, \lambda), \lambda)\right\|\right. \\
&+ \frac{1}{\gamma_{2}} \| g(x(\bar{\omega}, \lambda), y(\bar{\omega}, \lambda), \bar{\lambda}) \\
&-g(x(\bar{\omega}, \lambda), y(\bar{\omega}, \lambda), \lambda) \| \\
&+\frac{\rho_{2}}{\gamma_{2}} \| G(x(\bar{\omega}, \lambda), y(\bar{\omega}, \lambda), \bar{\lambda}) \\
&-G(x(\bar{\omega}, \lambda), y(\bar{\omega}, \lambda), \lambda) \| \\
&+\eta\|\lambda-\bar{\lambda}\|\},
\end{aligned}
$$

where $\kappa$ is defined by $(* * *)$.

(C) Prove that the conclusion of Theorem 3.3.

From (3.28) and (3.40), by the assumptions for $H_{1}, f, F, H_{2}, g$, and $G$ in Theorem 3.3 and relation

$$
\|x(\omega, \lambda)-x(\bar{\omega}, \bar{\lambda})\| \leq\|x(\omega, \lambda)-x(\bar{\omega}, \lambda)\|+\|x(\bar{\omega}, \lambda)-x(\bar{\omega}, \bar{\lambda})\|,
$$

we know that $x(\omega, \lambda)$ is continuous (or Lipschitz continuous).

From (3.29) and (3.39), by the assumptions for $H_{1}, f, F, H_{2}, g$ and $G$ in Theorem 3.3 and relation

$$
\|y(\omega, \lambda)-y(\bar{\omega}, \bar{\lambda})\| \leq\|y(\omega, \lambda)-y(\bar{\omega}, \lambda)\|+\|y(\bar{\omega}, \lambda)-y(\bar{\omega}, \bar{\lambda})\|,
$$

we know that $y(\omega, \lambda)$ is continuous (or Lipschitz continuous).

\section{Acknowledgment}

The author would like to thank the anonymous referee for reading this paper carefully, providing valuable suggestions and comments. The work was supported by the National Science Foundation of China (no. 10561007) and Science and Technology Research Project of Education Department in Jiangxi province (GJJ10269).

\section{References}

[1] R. P. Agarwal, N.-J. Huang, and M.-Y. Tan, "Sensitivity analysis for a new system of generalized nonlinear mixed quasi-variational inclusions," Applied Mathematics Letters, vol. 17, no. 3, pp. 345-352, 2004.

[2] R. P. Agarwal, Y. J. Cho, and N. J. Huang, "Sensitivity analysis for strongly nonlinear quasi-variational inclusions," Applied Mathematics Letters, vol. 13, no. 6, pp. 19-24, 2000.

[3] X. P. Ding, "Sensitivity analysis for generalized nonlinear implicit quasi-variational inclusions," Applied Mathematics Letters, vol. 17, no. 2, pp. 225-235, 2004. 
[4] S. Dafermos, "Sensitivity analysis in variational inequalities," Mathematics of Operations Research, vol. 13, no. 3, pp. 421-434, 1988.

[5] R. N. Mukherjee and H. L. Verma, "Sensitivity analysis of generalized variational inequalities," Journal of Mathematical Analysis and Applications, vol. 167, no. 2, pp. 299-304, 1992.

[6] M. A. Noor, "Sensitivity analysis for quasi-variational inequalities," Journal of Optimization Theory and Applications, vol. 95, no. 2, pp. 399-407, 1997.

[7] K. R. Kazmi and F. A. Khan, "Sensitivity analysis for parametric generalized implicit quasi-variationallike inclusions involving $p$ - $\eta$-accretive mappings," Journal of Mathematical Analysis and Applications, vol. 337, no. 2, pp. 1198-1210, 2008.

[8] R. U. Verma, "General system of $A$-monotone nonlinear variational inclusion problems with applications," Journal of Optimization Theory and Applications, vol. 131, no. 1, pp. 151-157, 2006.

[9] Y.-P. Fang and N.-J. Huang, "H-monotone operator and resolvent operator technique for variational inclusions," Applied Mathematics and Computation, vol. 145, no. 2-3, pp. 795-803, 2003. 


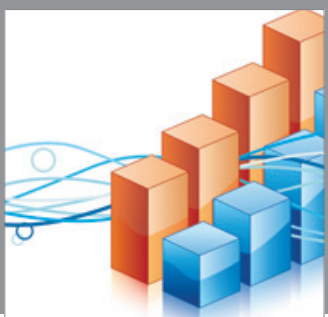

Advances in

Operations Research

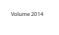

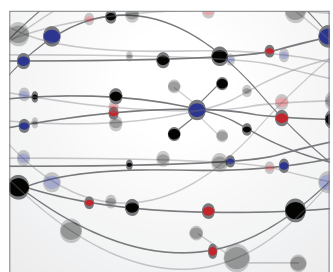

\section{The Scientific} World Journal
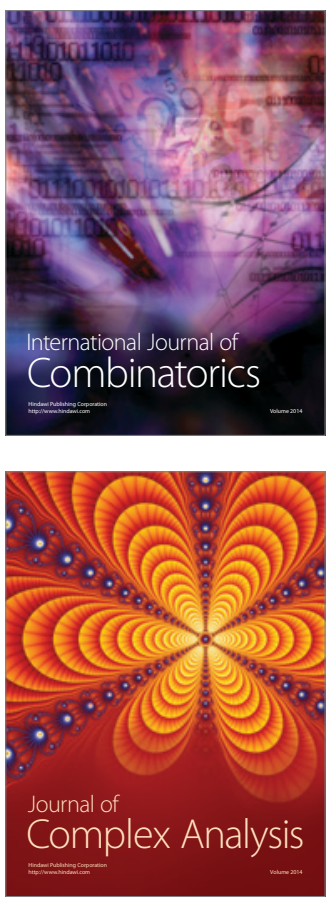

International Journal of

Mathematics and

Mathematical

Sciences
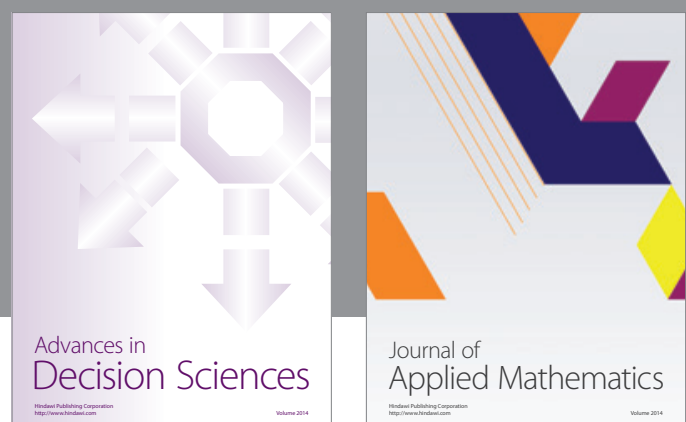

Journal of

Applied Mathematics
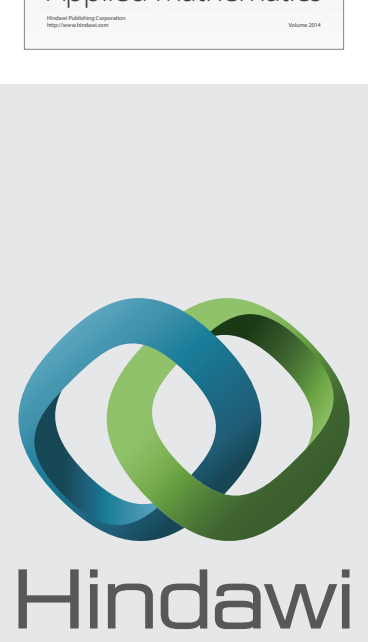

Submit your manuscripts at http://www.hindawi.com
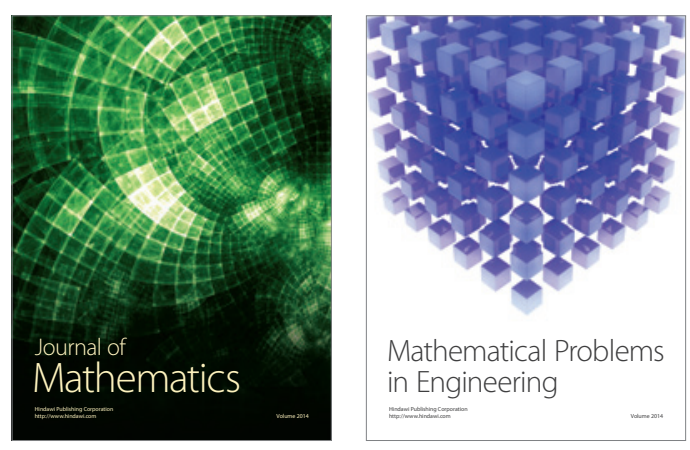

Mathematical Problems in Engineering
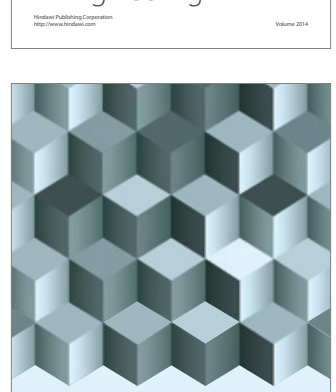

Journal of

Function Spaces
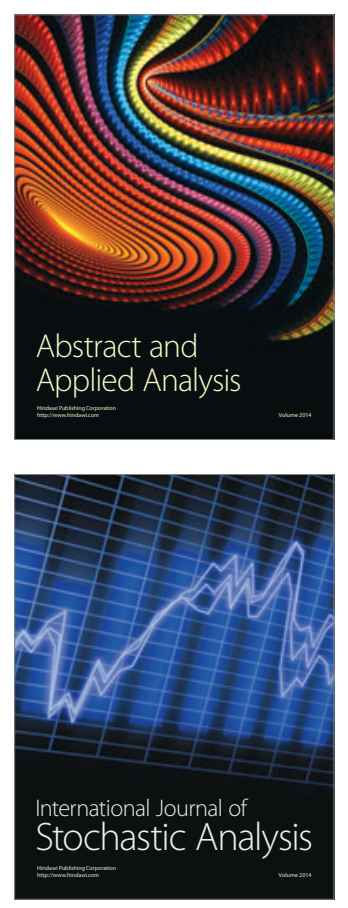

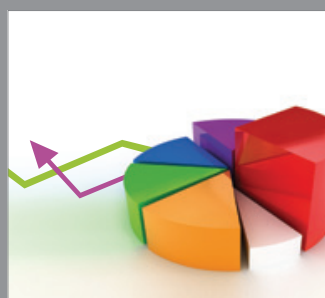

ournal of

Probability and Statistics

Promensencen
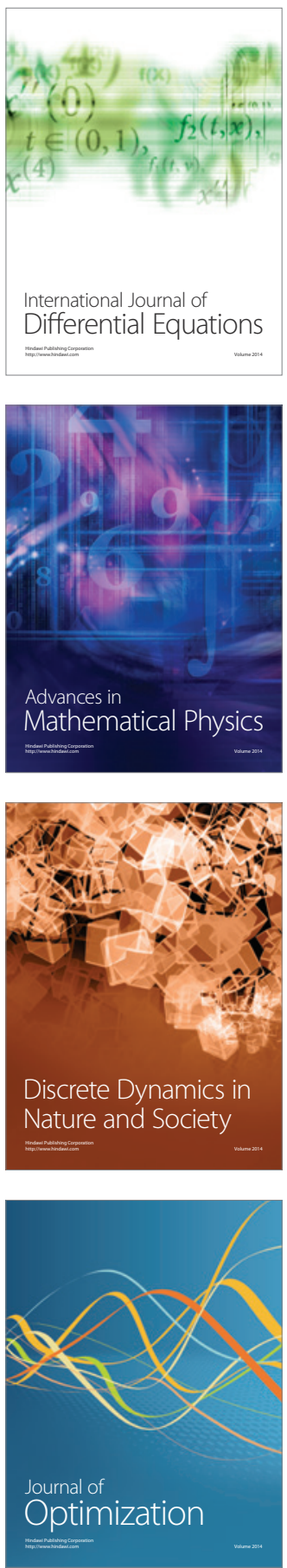